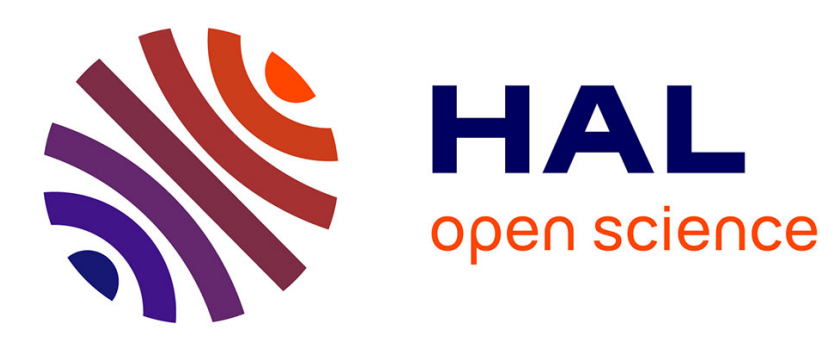

\title{
Temporal changes in the lunar soil from correlation of diffuse vibrations
}

\author{
Christoph Sens-Schönfelder, Éric Larose
}

\section{To cite this version:}

Christoph Sens-Schönfelder, Éric Larose. Temporal changes in the lunar soil from correlation of diffuse vibrations. Physical Review E: Statistical, Nonlinear, and Soft Matter Physics, 2008, 78, pp.045601. 10.1103/PhysRevE.78.045601 . hal-00325967

\section{HAL Id: hal-00325967 https://hal.science/hal-00325967}

Submitted on 30 Sep 2008

HAL is a multi-disciplinary open access archive for the deposit and dissemination of scientific research documents, whether they are published or not. The documents may come from teaching and research institutions in France or abroad, or from public or private research centers.
L'archive ouverte pluridisciplinaire HAL, est destinée au dépôt et à la diffusion de documents scientifiques de niveau recherche, publiés ou non, émanant des établissements d'enseignement et de recherche français ou étrangers, des laboratoires publics ou privés. 


\title{
Temporal changes in the lunar soil from correlation of diffuse vibrations
}

\author{
Christoph Sens-Schönfelder* \\ Institute for Geophysics and Geology, Universität Leipzig \\ Talstr. 35, 04103 Leipzig, Germany. \\ Eric Larose \\ Laboratoire de Géophysique Interne et Tectonophysique, \\ Univ. Joseph Fourier and CNRS, Grenoble France \\ BP 53, 38041 GRENOBLE cedex 9 France
}

(Dated: September 30, 2008)

\begin{abstract}
It was recently demonstrated that one can reconstruct the impulse response between passive sensors, by cross-correlating diffuse waves or ambient noise. Using seismic waves recorded on the moon, we show here that not only direct waves can be retrieved but also late arrivals that have been scattered before reaching the seismometers. As these late arrivals propagate for longer time, they are more sensitive to weak perturbations of the medium such as velocity changes. This high sensitivity of scattered waves is used to monitor periodic velocity changes in the lunar soil by measuring small delays of the passively retrieved coda waves. The velocity changes result from temperature variations due to periodic heating of the lunar surface by the sun.

PACS numbers:
\end{abstract}

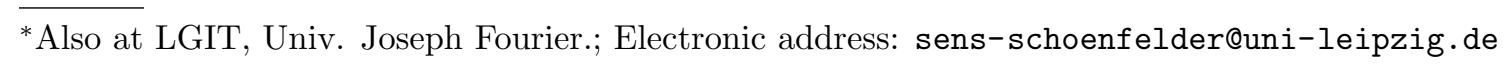


In earth and planetary sciences, very small amplitude phenomena sometimes accumulate over very long time to eventually build up a large dynamic process. For instance, displacements of the order of a few millimeters per year in the Earth's crust can accumulate over geological times (millions of years) to totally reshape the continents at a global scale. The understanding of the physics of the solid Earth and other planets thus relies on our ability to measure and analyze variations on time scales that are accessible to observation. This requires having (1) a perfectly reproducible experimental setup, and (2) very precise measurement. In seismology and seismic prospecting where variation of the seismic velocity is of primary interest, these two features have been an issue for decades. First, earthquakes are not repeatable (except certain seismic doublets [1]), and the energy released by controlled sources is limited. Second, the relative precision of conventional repeated travel-time measurements hardly attains $\delta v / v \approx 10^{-3}$, which is not sufficient to monitor dynamic processes in the Earth. In the following, we will apply a technique that allows to address these two issues simultaneously by taking advantage of the mesoscopic nature of seismic waves in a natural environment.

Mesoscopy loosely refers to all interference phenomena that occur in multiple scattering media. In this mesoscopic regime [2,3], we have $\lambda<\ell^{*} \ll \ell_{a}$ where $\lambda$ is the wavelength, $\ell^{\star}$ is the transport mean free path that corresponds to the distance that a wave must travel to forget its incident direction, and $\ell_{a}$ is the usual intrinsic absorption length. Since the early 80's, several mesoscopic phenomena have been observed, including (among many others) field correlation functions [4] and intensity fluctuations, acoustic time-reversal [5], coherent backscattering [6, 7] and more recently Anderson localization [8]. Today, the study of diffuse wave propagation has achieved an interdisciplinary character without precedent, and many kinds of waves are known to obey a diffusion equation: optical waves in clouds or biological tissues, electrons in quantum conductors, acoustic waves in granular media, to name only a few. Mesoscopic principles such as transport theory [9], equipartition [10] or weak localization [11] have also been fruitfully applied to seismic waves. These discoveries cleared the way for a new method in imaging [12]. The space-time correlations of the seismic coda or ambient noise yield the elastic Green's function of the local geophysical environment as if a source was placed at a receiver (passive imaging). This passively obtained Green's function in turn, provides us with precious information about Earth structure at depth that would have been very difficult to obtain actively, simply because powerful local sources are 
absent $[13,14]$. This principle can fix the first issue mentioned in the introduction: the idea is to take advantage of ambient vibrations to obtain a robust and repetitive estimate of the elastic impulse response of the medium at different dates.

The second limitation reported in the introduction (the precision of the measurement) can be overcome by taking advantage of the diffusive nature of coda waves in heterogeneous media. Because they travel very long paths, scattered waves are much more sensitive to weak variations of medium properties than direct waves. This idea has led to the to seismic "doublet" technique [1], and has recently undergone a vigorous revival under the name Coda Wave Interferometry [15]. This idea was also investigated in optics and in acoustics where it has led to the so-called Diffuse (Acoustic) Waves Spectroscopy technique $[16,17]$.

The possibility of mixing the two mesocopic principles of passive imaging and Diffuse Waves Spectroscopy has been subject to very recent pioneering investigations in seismology. We can report fascinating observations of weak velocity variations around volcanoes [18, 19] and active faults [20] that have been made with Passive Image Interferometry (PII). Nevertheless, we have to acknowledge that changes inferred from PII on the Earth could not yet be compared to direct observations and may have various causes ranging from meteorology to tectonics. The situation on the moon appears to be much better under control: the solar heating which is the most probable source of changes can be independently evaluated in a quantitative way. This is the primary motivation to work in this spacial natural laboratory. The purpose of the present paper is thus to determine the changes in the lunar crust and to show that they are generated by the solar heating. This allows to check the validity of the PII technique in a natural environment where the source of change is precisely known.

The properties of the seismic wave field on the moon are perfectly suited for the application of mesoscopic concepts. Pioneering works in the 70's have demonstrated the diffusive nature of seismic waves in the heterogeneous and highly fractured regolith. We can deduce from the envelopes of seismic records acquired with controlled sources that the shallow subsurface is highly scattering $\left(10 \mathrm{~m}<\ell^{\star}<100 \mathrm{~m}\right)$ but weakly attenuating $\left(\ell_{a} \sim 5 \mathrm{~km}\right)$ [21]. With a wavelength of the order of ten meters we work well in the range of mesoscopic regime. On the Earth, absorption is greater and scattering is weaker, which results in slower convergence of the correlations toward the Green's function [22]. Nevertheless PII will still be possible because we can compensate the feeble convergence by processing more data. 


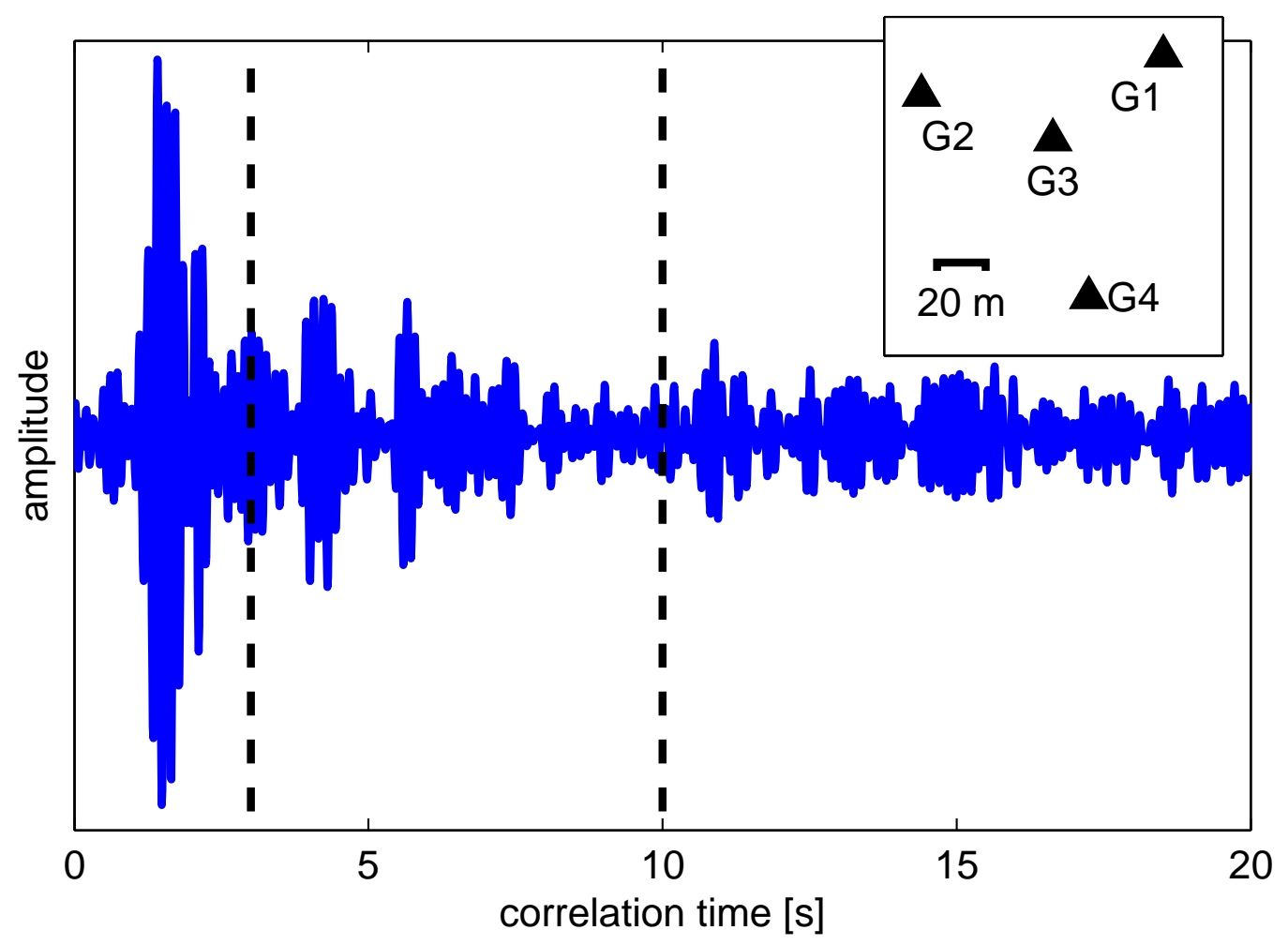

FIG. 1: NCF between geophones G2 and G3 retrieved from ten days of noise around sunset. Inset: map of the geophone array.

As part of the Lunar Seismic Profiling Experiment the Apollo 17 crew installed 4 geophones at the Taurus-Littrow landing site on the moon. The configuration of the triangular array installation is shown in the inset of figure 1. The geophones show a flat response between $3 \mathrm{~Hz}$ and $20 \mathrm{~Hz}$. From August 1976 until April 1977 the four geophones were continuously operated. In the present study we analyze this continuous dataset. As noted by Latham et al. [23] and Larose et al. [24] the ambient vibrations that are recorded on the moon are related to thermal moonquakes of which most are too small to be clearly detected. To verify this, the upper panel of figure 2 shows the noise level at the central station of the array. Noise level is defined here as the daily integral of the seismogram's envelope. The noise amplitude peaks shortly before sunset and has a second peak right after sunrise. This is the expected behavior for energy released from thermally stressed rocks that is related to temporal changes of temperature. The thermally generated seismic noise allows to retrieve approximations of Green's functions between seismic sensors. With the four geophones it is possible to retrieve approximations of ten Green's functions between all possible sensors, 

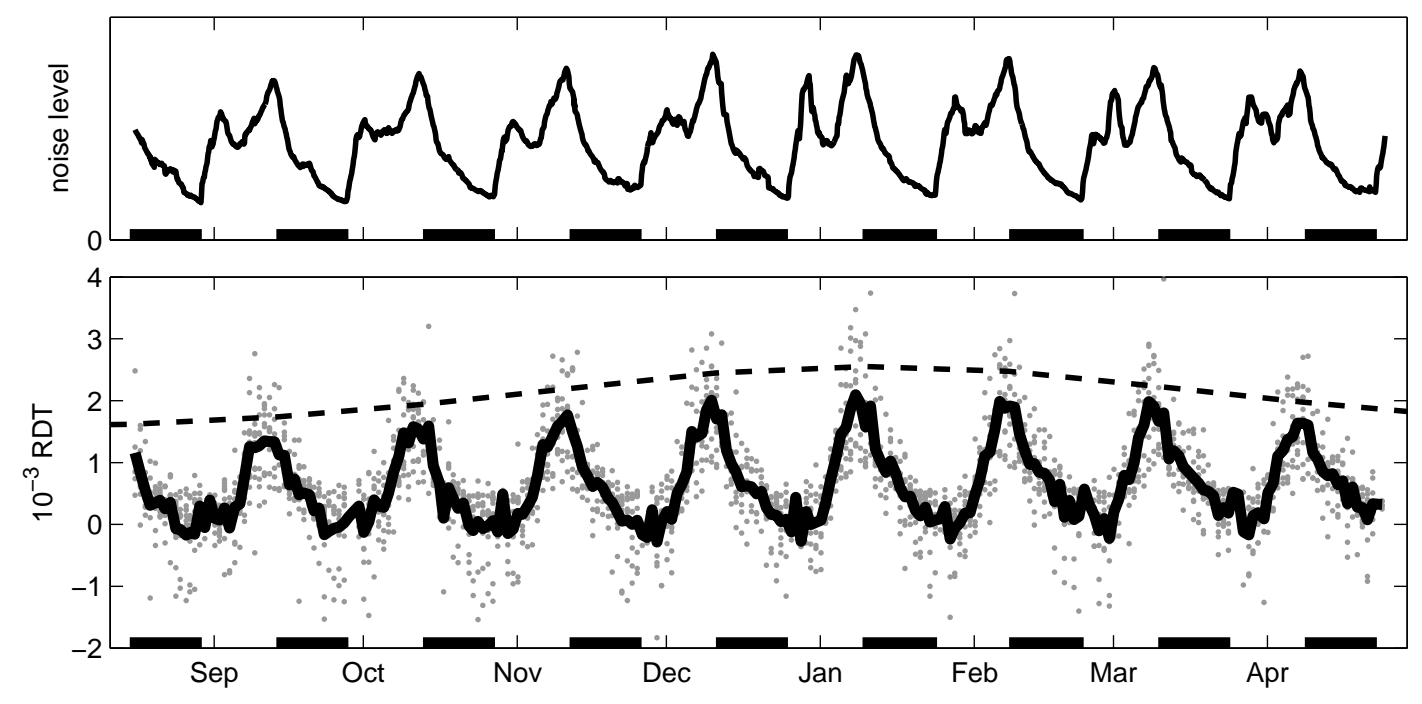

FIG. 2: Noise level and relative delay time (RDT) variations of seismic waves in the shallow lunar crust. Upper panel: Variations of the noise level at the central station G3. Lower panel: Gray dots: individual measurements of the RDT. Continuous curve: average over the ten available station configurations. Dashed curve: qualitative course of the inflow of thermal energy at lunar noon. Bold line segments at the bottom of each panel indicate lunar night

including auto-correlations. These noise correlation functions (NCF) are computed for the eight months of available data in segments of 24 hours. The records with a dynamic range of 7 bits are clipped at an amplitude of \pm 20 counts around the zero position. As an example of the correlation, we plot on figure 1 the NCF between geophones G2 and G3: a direct (Rayleigh) wave is reconstructed, followed by late arrivals scattered at surrounding heterogeneities. During propagation the seismic waves interact with the medium. In case of temporal velocity changes the seismic waves speed up or slow down compared to a reference state. If the relative velocity change is homogeneous in space, the resulting delay is proportional to the travel time of the wave: the relative delay time (RDT), i.e. the delay of a seismic phase divided by its travel time is constant.

Here the RDT is estimated as suggested by Sens-Schönfelder and Wegler [18]. The NCF is stretched by a factor $\epsilon=R D T+1$ and compared with a reference $\mathrm{NCF}_{r}$ by means of the correlation coefficient for travel times between $3 \mathrm{~s}$ and $10 \mathrm{~s}$. The $\epsilon$ that maximizes the 
correlation coefficient $C C$ :

$$
C C(\epsilon)=\frac{\int_{\tau=3 s}^{\tau=10 s} N C F(\tau \epsilon) N C F_{r}(\tau) d \tau}{\int_{\tau=3 s}^{\tau=10 s} N C F(\tau \epsilon)^{2} d \tau \int_{\tau=3 s}^{\tau=10 s} N C F_{r}(\tau)^{2} d \tau}
$$

gives the RDT according to the above equation. In doing so it is implicitly assumed that the heterogeneity of the velocity change is weak. If this is violated the paths of the waves are altered and the NCFs decorrelate. The bottom panel of figure 2 shows the RDT from all cross- and auto-correlations measured every 24 hours in the frequency band between $6 \mathrm{~Hz}$ and $11 \mathrm{~Hz}$. The average over the ten station configurations is shown by the continuous curve. Black line segments at the bottom of the graphs indicate lunar night. The RDT curve in figure 2 shows a clear periodicity of approximately one month similar to the noise level. These variations might be related to the position of the Earth (tidal effect with a periodicity of 27.3 days) or to the position of the sun (solar heating with a periodicity of 29.5 days). A thorough Fourier analysis favors the latter periodicity [26]. The mechanism for the influence of the sun on RDT of seismic waves on the moon is therefore thermal heating. We hypothesize that the changes of the temperature profile due to heating by the sun's radiation during lunar day reduces the seismic velocity, causing the variations of the RDT.

To support this hypothesis, a thermal modeling is performed that simulates the heat conduction in the lunar subsurface. Using the knowledge about heat conductivity in the shallow lunar crust, gained from the Apollo experiments, it is possible to reproduce the observed RDT variations under the following two assumptions. (1) The velocity variations are proportional to temperature variations. This is a first order approximation, but sufficient for the relevant temperature range. (2) The RDT is proportional to the average temperature change over $2 \mathrm{~m}$ depth. This is an acceptable assumption because the monthly temperature variations are limited to a very shallow layer above about $2 \mathrm{~m}$ depth [25]. The depth profiles of the thermal conductivity and density are taken from figure 9-10 of Langseth et al. [25]. This structure contains a $2 \mathrm{~cm}$ thick top layer in which heat is transfered not only by conduction but also by radiation. The heat flux through the surface is determined by the emission of thermal radiation (the Stefan-Boltzmann law) and by the inflow of energy by illumination from the sun. In doing so the model is completely determined by the changing incidence angle of the radiation from the sun, the sun-moon distance, the emission coefficient (1.0 for infrared radiation from the moon's surface), the absorption coefficient of the moon's 


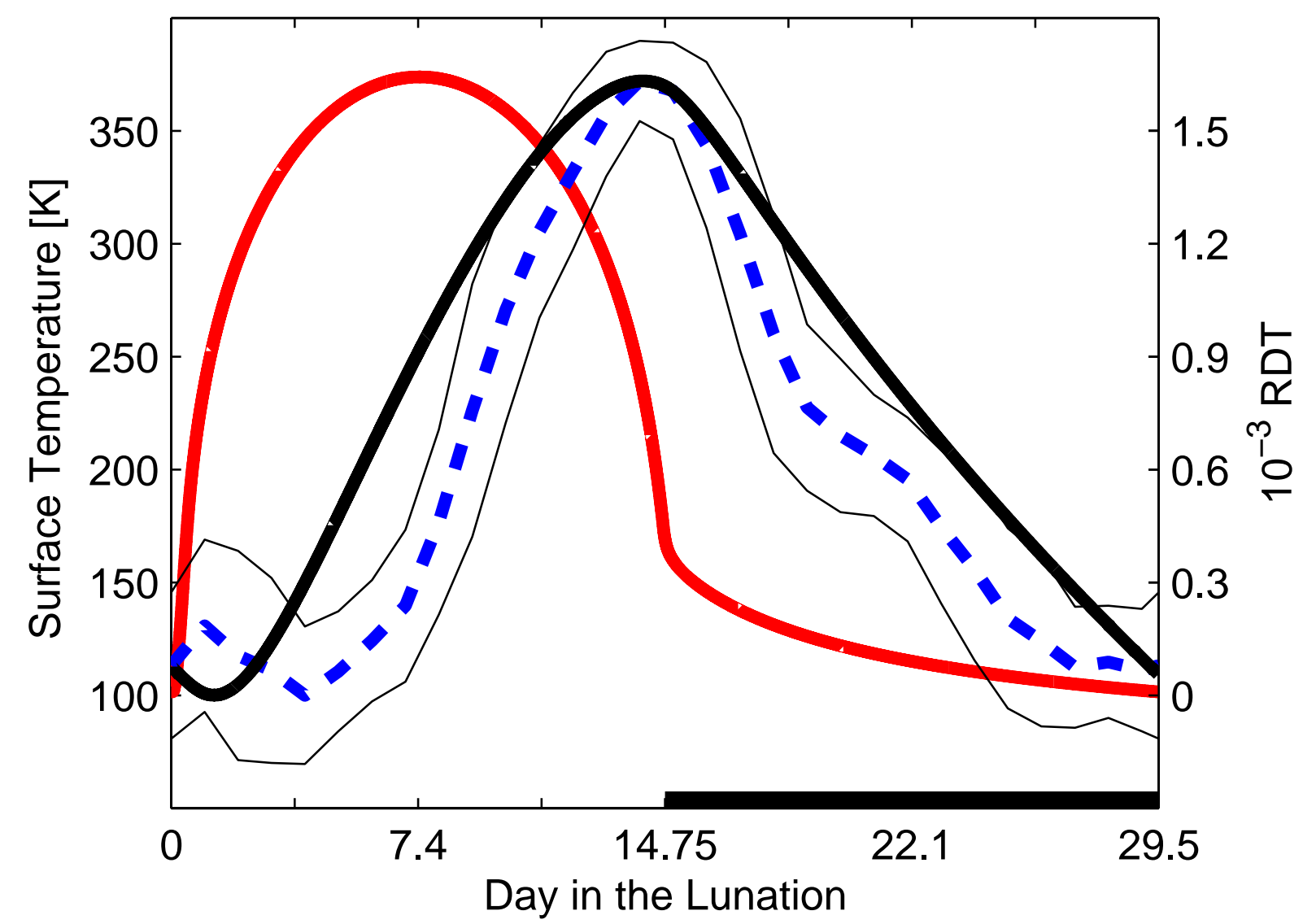

FIG. 3: Results of the thermal model calculations for one lunation from sunrise to sunrise. Dashed curve: stack of the measured relative delay time variations from the eight lunations. Thin lines indicate the standard deviation. Continuous curves show model predictions for the relative delay times (black) and surface temperature (gray / red).

surface for radiation from the sun (0.6), the specific heat $(600 \mathrm{~kJ} /(\mathrm{kg} \mathrm{K}))$ and the solar constant. The theoretical prediction for the RDT that derives from this simple model is shown by the black curve in figure 3. Measured RDT, averaged over eight different lunations, is in the dashed curve between the two thin lines that indicate one standard deviation. The shape of the resulting surface temperature curve from sunrise to sunrise is shown by the gray (red) line in figure 3, as measured by Langseth et al. [25]. The phase shift of the measured RDT with respect to the surface temperature is due to thermal diffusion, and is well reproduced by our simple model. The agreement between theoretical and measured RDT supports the hypothesis that the sun causes the RDT variations. The phase shift compared to the surface temperature curve excludes the possibility that the RDT variations 
are caused by technical effects due to heating of the instruments.

Another observation that supports a relation between the RDT variations and heating by the sun concerns the amplitudes of the peaks of the RDT. They vary systematically from one lunation to another. The peak in January 1977 is about $20 \%$ higher than the peak in September 1976 (see figure 2). This variability can be qualitatively explained by variations in the sun-moon distance and energy inflow due to the excentricity of the Earth's orbit. The qualitative course of the energy inflow at noon is shown by the dashed curve in figure 2 . It is in agreement with the changes of the RDT peak amplitudes.

Combining two concepts of mesoscopic wave physics we explore an almost historical dataset in a completely new way. Using 30 years old Apollo seismic data from the moon, we show that seismic waves in the crust are periodically slowed down. The shape of these delays and their periodicity indicate that they result from heating by the sun. Even variations of energy inflow due to changes in the sun-moon distance can be seen. The delay time variations reflect changes in the temperature profile of the lunar crust such that increased temperature lowers the seismic velocity. We propose a theoretical prediction for the temperature variation of the lunar subsurface that reproduces experimental variations. Our simple model is based on current knowledge of the thermal properties of the lunar surface layer. A detailed inversion of the delay time curves with respect to the parameters of the lunar material, i.e. conductivity, density, heat capacity and amount of radiative heat transport will add to our understanding of the heat transport in the lunar regolith. From a broader point of view, the experiment reported here demonstrates that we can not only reconstruct direct waves between two passive sensors with ambient noise (passive imaging), but also that we can take advantage of reconstructed diffuse paths to monitor very weak changes of a complex (and natural) environment.

This is an encouraging result not only for planetary seismology but also for further terrestial studies in rapidly changing environments like volcanoes and fault zones. The possibility to apply PII also in vibrating manmade structures of virtually all scales lets us expect various applications in structural health monitoring and life cycle management in the next years.

We wish to acknowledge L. Margerin for critical comments, and Y. Nakamura for pro- 
viding us with the data.

[1] G. Poupinet, W. L. Ellsworth, and J. Frechet, J. Geophys. Res. 89, 5719 (1984).

[2] P. Sheng, Introduction to wave scattering, localization and mesoscopique phenomena (Academic Press, 1995).

[3] E. Akkermans and G. Montambaux, Mesoscopic Physics of Electrons and Photons (Cambridge University press, 2007).

[4] R. Pnini and B. Shapiro, Phys. Rev. B 39, 6986 (1989).

[5] A. Derode, P. Roux, and M. Fink, Phys. Rev. Lett. 75, 4206 (1995).

[6] M. P. Van Albada and A. Lagendijk, Phys. Rev. Lett. 55, 2692 (1985).

[7] P.-E. Wolf and G. Maret, Phys. Rev. Lett. 55, 2696 (1985).

[8] M. Störzer, P. Gross, C. M. Aegerter, and G. Maret, Phys. Rev. Lett. 96, 063904 (2006).

[9] L. Margerin, M. Campillo, and B. A. van Tiggelen, Geophys. J. Int. 134, 596 (1998).

[10] R. Hennino, N. Trégourès, N. M. Shapiro, L. Margerin, M. Campillo, B. A. van Tiggelen, and R. L. Weaver, Phys. Rev. Lett. 86, 3447 (2001).

[11] E. Larose, L. Margerin, B. A. van Tiggelen, and M. Campillo, Phys. Rev. Lett. 93, 048501 (2004).

[12] R. L. Weaver and O. I. Lobkis, Phys. Rev. Lett. 87, 134301 (2001).

[13] M. Campillo and A. Paul, Science 299, 547 (2003).

[14] N. M. Shapiro, M. Campillo, L. Stehly, and M. H. Ritzwoller, Science 307, 1615 (2005).

[15] R. Snieder, A. Grêt, H. Douma, and J. Scales, Science 295, 2253 (2002).

[16] D. J. Pine, D. A. Weitz, P. M. Chaikin, and E. Herbolzheimer, Phys. Rev. Lett. 60, 1134 (1988).

[17] M. L. Cowan, I. P. Jones, J. H. Page, and D. A. Weitz, Phys. Rev. E 65, 66605 (2002).

[18] C. Sens-Schönfelder and U. C. Wegler, Geophys. Res. Lett. 33, L21302 (2006).

[19] F. Brenguier, N. M. Shapiro, M. Campillo, V. Ferrazzini, Z. Duputel, O. Coutant, and A. Nercessian, Nature geosciences 1, 126 (2008).

[20] U. Wegler and C. Sens-Schoenfelder, Geophys. J. Int. 168, 1029 (2007).

[21] A. M. Dainty and M. N. Toksoz, Phys. Earth Planet. Inter. 26, 250 (1981).

[22] E. Larose, and A. Derode, J. Appl. Phys. 103, (2008). 
[23] G. Latham, M. Ewing, J. Dorman, Y. Nakamura, F. Press, N. Toksoz, G. Sutton, F. Duennebier, and D. Lammlein, Moon 7, 396 (1973).

[24] E. Larose, A. Khan, Y. Nakamura, and M. Campillo, Geophys. Res. Lett. 32, L16201 (2005).

[25] M. G. Langseth, S. J. Keihm, and J. L. Chute, Apollo 17 Preliminary Science Report, [NASA SP-330] p. 9.1 (1973).

[26] See EPAPS Document No. XXXX for the detailed spectrum. For more information on EPAPS, see http://www.aip.org/pubservs/epaps.html. 